
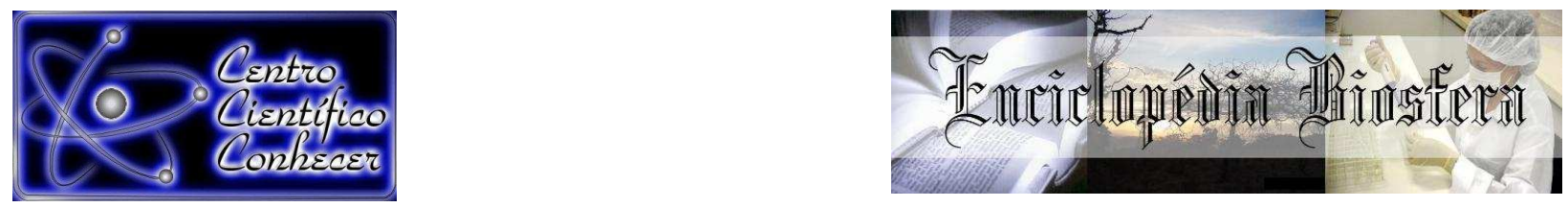

\title{
SOBREVIVÊNCIA E DESENVOLVIMENTO DE ESPÉCIES NATIVAS EM PLANTIO DE RECUPERAÇÃO DA MATA ATLÂNTICA AOS VINTE MESES DE IDADE EM VIÇOSA, MINAS GERAIS
}

Vicente Toledo Machado de Morais Junior ${ }^{1}$, Samuel José Silva Soares da Rocha ${ }^{1}$, Indira Bifano Comini ${ }^{1}$, Eliana Boaventura Bernardes Moura Alves ${ }^{2}$, Laércio Antônio Gonçalves Jacovine ${ }^{3}$

${ }^{1}$ Mestrando(a) em Ciência Florestal no Departamento de Engenharia Florestal da Universidade Federal de Viçosa, Viçosa, Minas Gerais, Brasil

(vicente.moraisjr@gmail.com)

2Doutoranda em Ciência Florestal no Departamento de Engenharia Florestal da Universidade Federal de Viçosa, Viçosa, Minas Gerais, Brasil

3Professor Assistente no Departamento de Engenharia Florestal da Universidade Federal de Viçosa, Viçosa, Minas Gerais, Brasil

Recebido em: 08/04/2016 - Aprovado em: 30/05/2016 - Publicado em: 20/06/2016 DOI: 10.18677/Enciclopedia_Biosfera_2016_110

\begin{abstract}
RESUMO
Objetivou-se avaliar a sobrevivência e o crescimento de espécies nativas, no período de 20 meses, em plantio de recuperação de uma área degradada em Viçosa, Minas Gerais. O estudo foi conduzido na área denominada Espaço Aberto de Eventos da Universidade Federal de Viçosa (UFV), em Viçosa, Minas Gerais. O plantio foi realizado em dezembro de 2013, em uma área de 0,25 ha, com combinação de espécies florestais nativas da Mata Atlântica. Foram plantadas 500 mudas, no espaçamento $2 \mathrm{~m} \times 2 \mathrm{~m}$, em Delineamento em Blocos Casualizados (DBC), sendo cada bloco constituído de 25 espécies. Foram mensuradas a altura e o diâmetro ao nível do solo. Observou-se um índice geral de sobrevivência de $50,4 \%$. Dentre as espécies analisadas, a Schinus terebinthifolius, Sapindus saponaria, Plathymenia reticulata, Cassia grandis e Senna multijuga são recomendadas para plantios mistos de recuperação de áreas degradadas em situações similares à do experimento. As espécies Plathymenia reticulata e Senna multijuga, além do sucesso de sobrevivência, tiveram destaque nos parâmetros de crescimento. Assim, o estudo possibilita inferir a respeito da potencialidade e restrição de espécies florestais no plantio de recuperação da área degradada na Mata Atlântica.
\end{abstract}

PALAVRAS-CHAVE: Áreas degradadas, Crescimento, Plantio misto

\section{SURVIVAL AND DEVELOPMENT OF NATIVE SPECIES IN A RECOVERY PLANTING OF ATLANTIC FOREST AT TWENTY MONTHS OF AGE, IN VIÇOSA, MINAS GERAIS}

ABSTRACT
It's aimed to evaluate the survival and growth of native species in the period of 20
months, in a recovering planting of a degraded area in Viçosa, Minas Gerais. The 
study was conducted in the area called Open Space Events of the Federal University of Viçosa (UFV), Viçosa, Minas Gerais. The planting was carried out in December 2013 in an area of 0.25 ha, with combination of native species of the Atlantic Forest. 500 seedlings were planted, spaced $2 \mathrm{~m} \times 2 \mathrm{~m}$, in a randomized block design (RBD), with each block consisting of 25 species. Were measured the height and the diameter at the ground level. There was an overall survival rate of $50.4 \%$. Among the analyzed species, Schinus terebinthifolius, Sapindus saponaria, Plathymenia reticulata, Cassia grandis and Senna multijuga are recommended for recovery mixed plantings of degraded areas in similar situations to the experiment. The species Plathymenia reticulata and Senna multijuga, beyond the survival success, were highlighted in growth parameters. Thus, the study makes it possible to make inferences about the potential and restriction of forest species in the recovery planting of degraded area in the Atlantic Forest.

KEYWORDS: Degraded areas, Growth, Mixed planting

\section{INTRODUÇÃO}

A cobertura atual da Mata Atlântica brasileira é constituída por remanescentes do bioma que representam cerca de $12 \%$ de sua cobertura original em relação à que existia desde a época em que os colonizadores europeus ocuparam o território nacional (SOS MATA ATLÂNTICA, 2016).

Somente no período de 2013 a 2014, o desmatamento registrado foi de 18,2 mil ha. O estado de Minas Gerais foi responsável pela perda de 5.608 ha de Mata Atlântica, e ainda apresentou quatro dos dez municípios que mais desmataram em todo o bioma. Atualmente, restam apenas 2,8 milhões de hectares de Mata Atlântica em Minas Gerais, aproximadamente $10,3 \%$ da cobertura original do estado (SOS MATA ATLÂNTICA, 2015).

A Lei Federal no 9.985 de 2000, que instituiu o Sistema Nacional de Unidades de Conservação da Natureza, define a recuperação como a restituição de um ecossistema ou de uma população silvestre degradada a uma condição não degradada, que pode ser diferente de sua condição original (BRASIL, 2000). Segundo TABARELLI et al. (2010), iniciativas de recuperação de florestas tropicais são de extrema importância, pois objetivam o cumprimento da legislação ambiental, o restabelecimento de serviços ecossistêmicos e a proteção de espécies nativas. Por meio desses benefícios, paisagens muito antropizadas passam a ter seus serviços de conservação da biodiversidade potencializados.

A regeneração natural, em alguns casos, pode ser inexistente ou muito lenta, necessitando de intervenção humana para a área retornar a um estado biológico estável. Dessa forma, a regeneração artificial por meio de plantio de mudas, surge como alternativa na recuperação dessas áreas (QUEIROZ et al., 2013). Assim, a seleção de espécies mais adaptadas às condições de solos degradados constitui uma etapa fundamental na revegetação de ambientes em solos pobres. No entanto, de acordo com PAIVA (2000), ainda são incipientes os estudos que tratam do desempenho de espécies arbóreas no campo em recuperação de áreas degradadas.

Dessa forma, metodologias que contemplem o estudo do comportamento das espécies florestais em ambientes perturbados tornam-se essenciais, uma vez que o sucesso da recuperação da área está relacionado com a escolha adequada das espécies (FERREIRA et al, 2007). Nesse sentido, estudos relacionados ao desenvolvimento de espécies nativas em plantios mistos tornam-se importantes para 
subsidiar, dentre outros aspectos, a elaboração de planos de recuperação de áreas degradadas.

É nesse contexto que se insere a relevância desse trabalho, uma vez que por meio do estudo do crescimento e desenvolvimento de distintas espécies, pretendese gerar parâmetros que permitam melhorar a eficiência de projetos de recuperação de áreas degradadas, especialmente na Mata Atlântica. Assim, objetivou-se avaliar a sobrevivência e o crescimento inicial em diâmetro e altura de espécies nativas em plantio de recuperação de uma área degradada em Viçosa, Minas Gerais.

\section{MATERIAL E MÉTODOS}

O estudo foi conduzido na área denominada Espaço Aberto de Eventos da Universidade Federal de Viçosa (UFV), em Viçosa, Minas Gerais (coordenadas UTM: $721100 \mathrm{~m}-721250 \mathrm{~m}$ e $7702912 \mathrm{~m}-7703056 \mathrm{~m}$ ). O mapa da área foi confeccionado a partir de uma imagem do satélite Ikonos com resolução espacial de $1 \mathrm{~m}$, previamente ortorretificada (Figura 1).

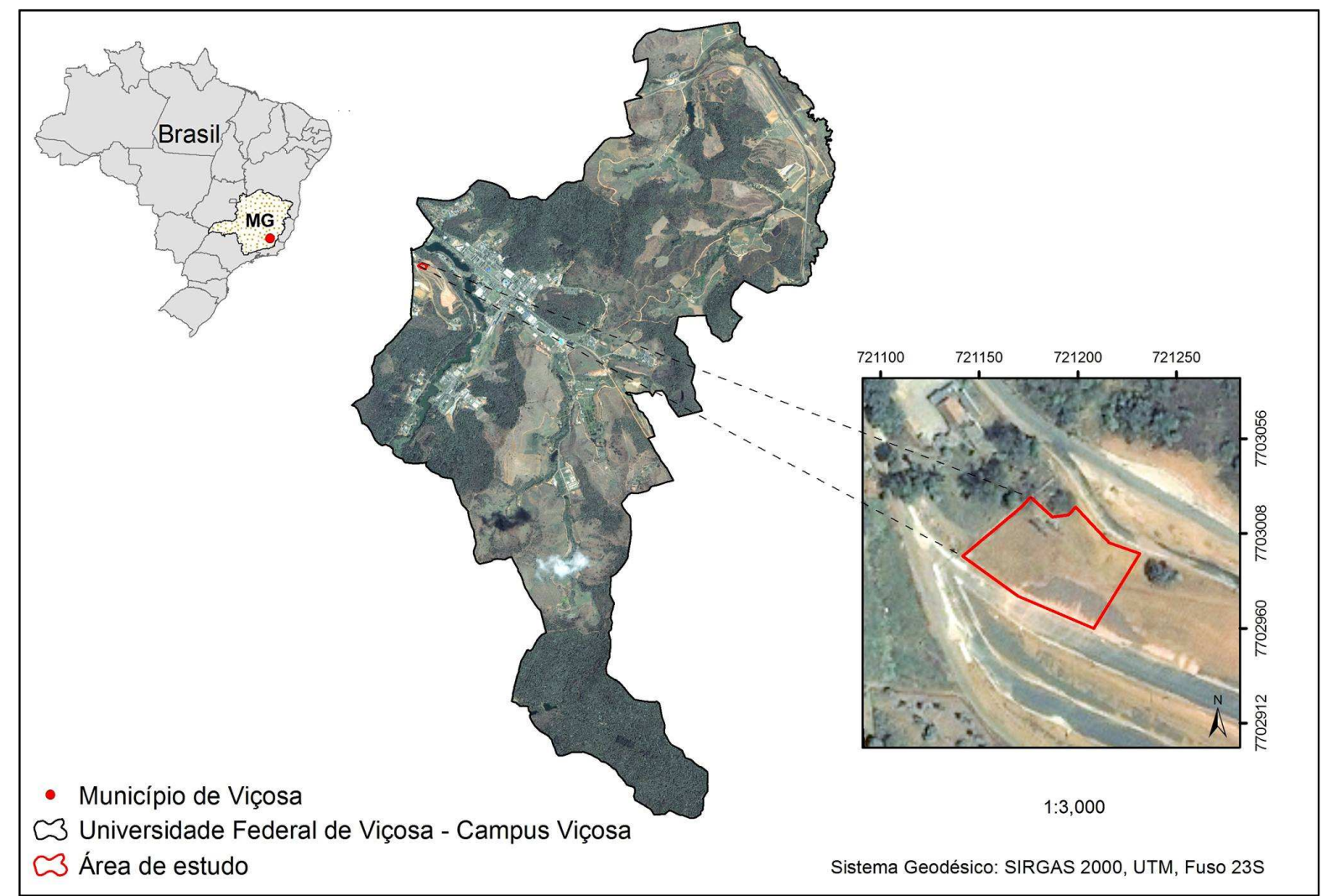

FIGURA 1: Localização da área de estudo.

Na região predominam solos do tipo Latossolo Vermelho e Amarelo Álico nos topos dos morros e Podzólicos Vermelho-Amarelo Câmbico nos terraços, em relevo caracterizado por colinas com a forma de espigões, muito seccionadas pela rede de drenagem natural e altitudes que variam de 650 a 800 m (ARRUDA et al., 1999; MEIRA-NETO \& MARTINS, 2002).

O clima na região é do tipo Cwa, segundo o sistema de Köppen, ou seja, mesotérmico com verões quentes e chuvosos e invernos frios e secos. Para o período de análise do estudo, dezembro de 2013 e agosto de 2015, a média de temperatura foi de $20,5{ }^{\circ} \mathrm{C}$ e a precipitação acumulada foi de $1.413,8 \mathrm{~mm}$ (UFV, 2016). 
O plantio foi realizado em dezembro de 2013, em uma área de 0,25 ha, com combinação de espécies florestais nativas da Mata Atlântica. Foram plantadas 500 mudas, no espaçamento $2 \mathrm{~m} \times 2 \mathrm{~m}$, em Delineamento em Blocos Casualizados (DBC), sendo cada bloco constituído de 25 espécies.

Uma vez que a área de plantio era recoberta por braquiária (Urochola decumbens (Stapf) R.D. Webster), realizou-se roçada antes de efetuar o plantio com o intuito de diminuir a competição.

As covas foram abertas nas dimensões de $0,30 \mathrm{~m} \times 0,30 \mathrm{~m} \times 0,30 \mathrm{~m}$ e adicionou-se $300 \mathrm{~g}$ de Superfosfato Simples $\left(18 \% \mathrm{P}_{2} \mathrm{O}_{5}\right)$ por cova. Após trinta dias, realizou-se a adubação de cobertura utilizando $100 \mathrm{~g}$ de NPK na formulação 20-5-20 por planta. A distribuição foi realizada em formato de círculo ao redor da muda, na projeção da copa das mesmas.

Periodicamente, foi realizado monitoramento da área para controle de formigas cortadeiras, coroamento das mudas e controle de mato competição.

A análise da sobrevivência e do crescimento de cada espécie seguiu metodologia adaptada de OLIVEIRA et al. (2015), de acordo com as equações propostas a seguir.

$$
I S_{j}(\%)=\left(N_{f_{j}} / N_{0_{j}}\right) \times 100
$$

Em que:

IS $S_{j}(\%)$ : Índice de sobrevivência da $j$-ésima espécie (percentual);

$N_{f_{j}}$ : Número de indivíduos sobreviventes da j-ésima espécie;

$N_{0_{j}}$ : Número inicial de indivíduos plantados da j-ésima espécie.

O crescimento foi avaliado a partir do Incremento Médio Mensal (IMM), em altura $\left(\mathrm{cm} \cdot \mathrm{mês}^{-1}\right)$ e em diâmetro $\left(\mathrm{mm} \cdot \mathrm{mês}^{-1}\right)$.

$$
I M M_{H_{j}}=\frac{1}{I N_{j}} \times \sum_{i=1}^{n}\left(H_{f_{i j}}-H_{0_{i j}}\right)
$$

Em que:

$I M M_{H_{j}}:$ Incremento médio mensal em altura da j-ésima espécie (cm.mês ${ }^{-1}$ );

I: Idade do plantio (meses);

$N_{j}$ : Número de indivíduos da $j$-ésima espécie;

$H_{f_{i j}}$ : Altura final do i-ésimo indivíduo da j-ésima espécie $(\mathrm{cm})$;

$H_{0_{i j}}$ : Altura inicial do i-ésimo indivíduo da j-ésima espécie $(\mathrm{cm})$.

$$
I M M_{D_{j}}=\frac{1}{I N_{j}} \times \sum_{i=1}^{n}\left(D_{f_{i j}}-D_{0_{i j}}\right)
$$

Em que:

$I M M_{D_{i}}:$ Incremento médio mensal em diâmetro da j-ésima espécie (mm.mês $\left.{ }^{1}\right)$; 
I: Idade do plantio (meses);

$N_{j}$ : Número de indivíduos da j-ésima espécie;

$D_{f_{i j}}$ : Diâmetro final do i-ésimo indivíduo da j-ésima espécie $(\mathrm{mm})$;

$D_{0_{i j}}$ : Diâmetro inicial do i-ésimo indivíduo da j-ésima espécie (mm).

A altura foi mensurada utilizando-se fita métrica graduada, em centímetros e o diâmetro ao nível do solo utilizando-se um paquímetro digital de precisão, em milímetros.

\section{RESULTADOS E DISCUSSÃO}

A partir do levantamento realizado, observou-se um índice geral de sobrevivência de 50,4\% para as mudas aos 20 meses de idade (Tabela 1).

TABELA 1: Índice de sobrevivência (\%) das espécies na área em recuperação, em Viçosa, MG

\begin{tabular}{l|c}
\hline \multicolumn{1}{c|}{ Nome científico } & IS (\%) \\
\hline Schinus terebinthifolius Raddi & 95,00 \\
\hline Sapindus saponaria L. & 95,00 \\
\hline Plathymenia reticulata Benth. & 90,00 \\
\hline Cassia grandis L. f. & 80,00 \\
\hline Senna multijuga (Rich.) H.S. Irwin \& Barneby & 80,00 \\
\hline Handroanthus chrysotrichus (Mart. ex DC.) Mattos & 75,00 \\
\hline Joannesia princeps Vell. & 65,00 \\
\hline Colubrina glandulosa Perkins & 65,00 \\
\hline Pachira glabra Pasq. & 65,00 \\
\hline Senna macranthera (DC. ex Collad.) H.S. Irwin \& Barneby & 60,00 \\
\hline Schizolobium parahyba (Vell.) Blake & 60,00 \\
\hline Hymenaea courbaril L. & 55,00 \\
\hline Anadenanthera peregrina (L.) Speg. & 50,00 \\
\hline Lecythis pisonis Cambess. & 50,00 \\
\hline Cedrela fissilis Vell. & 50,00 \\
\hline Citharexylum myrianthum Cham. & 45,00 \\
\hline Psidium cattleianum Sabine & 45,00 \\
\hline Sterculia apetala (Jacq.) H. Karst. & 40,00 \\
\hline Ceiba speciosa (A. St.-Hil.) Ravenna & 30,00 \\
\hline Poincianella pluviosa var. peltophoroides (Benth.) L. P. Queiroz & 20,00 \\
\hline Cariniana legalis (Mart.) Kuntze & 15,00 \\
\hline Tibouchina granulosa (Desr.) Cogn. & 10,00 \\
\hline Adenanthera pavonina L. & 10,00 \\
\hline Syzygium malaccense (L.) Merr. \& L.M. Perry & 5,00 \\
\hline Samanea inopinata (Harms) Barneby \& J.W. Grimes & 5,00 \\
\hline \multicolumn{1}{c}{ Média } & $\mathbf{5 0 , 4 0}$ \\
\hline
\end{tabular}

Em estudos de recuperação conduzidos na Mata Atlântica, MORAES et al. (2005) obtiveram um índice geral de sobrevivência de $90 \%$ para plantios mistos aos 48 meses de idade e CAMPOE et al. (2014) um índice de 92,1\% para plantios ENCICLOPÉDIA BIOSFERA, Centro Científico Conhecer - Goiânia, v.13 n.23; p.1255 
mistos aos 30 meses. Ambos valores são superiores à média geral encontrada nesse estudo.

Por outro lado, os resultados encontrados neste trabalho apresentaram-se superiores quando comparados a dois modelos avaliados no estudo de RESENDE et al. (2015). Os autores avaliaram o desenvolvimento silvicultural e a sobrevivência das espécies em diferentes modelos de plantio de mudas para recuperação da área do lixão de Inconfidentes, Minas Gerais. Para o modelo referente ao plantio de mudas de espécies arbóreas nativas, os autores encontraram uma sobrevivência geral de $28,33 \%$ aos 20 meses e $29,67 \%$ para o modelo com plantio de mudas de leguminosas arbóreas, valores bastante inferiores à média geral do plantio em questão.

Dentre as 25 espécies, 20\% apresentaram índice de sobrevivência superior a 80\%: S. terebinthifolius, S. saponaria, $P$. reticulata, $C$. grandis e S. multijuga.

No estudo de CAMPOE et al. (2014), S. terebinthifolius também se destacou em relação a esse parâmetro, com ausência de mortalidade. Para CARVALHO (2003), o sucesso da $S$. terebinthifolius é explicado pelo fato dessa espécie ser rústica e agressiva.

No estudo supracitado de RESENDE et al. (2015), os autores avaliaram, dentre outras espécies, $S$. terebinthifolius, $S$. parahyba e $S$. multijuga. No modelo de plantio de mudas de espécies arbóreas nativas, $S$. terebinthifolius também destacou-se em termos de sobrevivência (66\%). Já analisando o modelo de plantio de mudas de leguminosas arbóreas, os autores encontraram uma sobrevivência de aproximadamente $33 \%$ para $S$. parahyba e ausência de sobrevivência para $S$. multijuga. Essas últimas apresentaram sobrevivência de $40 \%$ e $43 \%$, respectivamente, quando consorciadas com a gramínea Chrysopogon zizanioides (L.) Roberty. Esses valores foram inferiores aos encontrados no presente trabalho, uma vez que a sobrevivência para as três espécies citadas foram de $95 \%, 60 \%$ e $80 \%$, respectivamente.

Dentre as demais espécies, $H$. courbaril (55\%), C. fissilis (50\%) e C. speciosa (30\%) apresentaram índices de sobrevivência inferiores aos verificados por CAMPOE et al. (2014), cujas sobrevivências foram de $100 \% ; 96,4 \%$ e $75 \%$, respectivamente. Já PEREIRA et al. (2012) obtiveram valor similar para índice de sobrevivência de $H$. courbaril $(66 \%)$ em estudo de plantios de recuperação em área degradada aos 18 meses em Uberlândia, Minas Gerais.

Em relação à análise dos parâmetros de crescimento no ano 2015, em termos de diâmetro destacam-se as espécies $C$. speciosa e $T$. granulosa e, em relação à altura, a S. parahyba e S. multijuga (Tabela 2).

TABELA 2: Média de crescimento em altura $(H$, em $\mathrm{cm})$ e diâmetro ( $D$, em mm), em 2013 e 2015, das espécies em crescimento na área de recuperação em Viçosa, MG

\begin{tabular}{l|c|c|c|c}
\hline \multicolumn{1}{c|}{ Nome científico } & $\mathbf{D}_{\mathbf{2 0 1 3}}$ & $\mathbf{H}_{\mathbf{2 0 1 3}}$ & $\mathbf{D}_{\mathbf{2 0 1 5}}$ & $\mathbf{H}_{\mathbf{2 0 1 5}}$ \\
\hline Adenanthera pavonina L. & 4,09 & 18,00 & 17,21 & 117,00 \\
\hline Anadenanthera peregrina (L.) Speg. & 4,08 & 33,55 & 45,87 & 223,70 \\
\hline Cariniana legalis (Mart.) Kuntze & 5,75 & 29,00 & 8,77 & 33,67 \\
\hline Cassia grandis L. f. & 5,05 & 36,69 & 25,59 & 127,13 \\
\hline Cedrela fissilis Vell. & 10,39 & 38,44 & 47,97 & 73,11 \\
\hline Ceiba speciosa (A. St.-Hil.) Ravenna & 8,84 & 65,75 & 128,36 & 334,33 \\
\hline Citharexylum myrianthum Cham. & 8,15 & 45,61 & 22,35 & 75,22 \\
\hline
\end{tabular}


Cont. Tab 2

\begin{tabular}{l|c|c|c|c}
\hline Colubrina glandulosa Perkins & 5,07 & 25,19 & 42,26 & 194,53 \\
\hline Handroanthus chrysotrichus (Mart. ex DC.) Mattos & 3,70 & 17,43 & 34,88 & 164,73 \\
\hline Hymenaea courbaril L. & 7,01 & 37,23 & 17,12 & 62,82 \\
\hline Joannesia princeps Vell. & 12,33 & 40,15 & 41,16 & 123,31 \\
\hline Lecythis pisonis Cambess. & 8,79 & 35,8 & 15,82 & 62,42 \\
\hline Pachira glabra Pasq. & 19,57 & 25,64 & 53,26 & 88,69 \\
\hline Plathymenia reticulata Benth. & 6,09 & 34,75 & 80,29 & 266,22 \\
\hline $\begin{array}{l}\text { Poincianella pluviosa var. peltophoroides (Benth.) L. P. } \\
\text { Queiroz }\end{array}$ & 6,48 & 19,25 & 39,92 & 143,25 \\
\hline Psidium cattleianum Sabine & 5,63 & 36,11 & 17,57 & 55,33 \\
\hline Samanea inopinata (Harms) Barneby \& J.W. Grimes & 6,08 & 42,00 & 7,59 & 30,00 \\
\hline Sapindus saponaria L. & 5,55 & 21,39 & 22,70 & 91,00 \\
\hline Schinus terebinthifolius Raddi & 8,02 & 58,42 & 62,21 & 219,05 \\
\hline Schizolobium parahyba (Vell.) Blake & 9,42 & 60,04 & 97,56 & 352,54 \\
\hline Senna macranthera (DC. ex Collad.) H.S. Irwin \& Barneby & 3,70 & 26,29 & 67,08 & 258,58 \\
\hline Senna multijuga (Rich.) H.S. Irwin \& Barneby & 4,93 & 47,25 & 96,65 & 341,81 \\
\hline Sterculia apetala (Jacq.) H. Karst. & 11,63 & 34,94 & 44,32 & 117,5 \\
\hline Syzygium malaccense (L.) Merr. \& L.M. Perry & 6,81 & 28,00 & 25,81 & 83,00 \\
\hline Tibouchina granulosa (Desr.) Cogn. & 6,11 & 62,00 & 112,60 & 157,00 \\
\hline \multicolumn{1}{|c|}{ Média } & $\mathbf{7 , 3 3}$ & $\mathbf{3 6 , 7 6}$ & $\mathbf{4 6 , 9 9}$ & $\mathbf{1 5 1 , 8 4}$ \\
\hline
\end{tabular}

No estudo de RESENDE et al. (2015), S. terebinthifolius apresentou altura superior a $2,75 \mathrm{~m}$ e o diâmetro do caule ao nível do solo de aproximadamente 55 $\mathrm{mm}$. Essa espécie, no presente estudo, destacou-se em termos de sobrevivência, obteve altura média de $219,05 \mathrm{~cm}$ e diâmetro médio de $62,21 \mathrm{~mm}$.

Nesse mesmo estudo, $S$. parahyba alcançou alturas aproximadas de 0,65 $\mathrm{m}$ e 1,30 $\mathrm{m}$, sem e com o consórcio com a gramínea, respectivamente, além de diâmetros de $21 \mathrm{~mm}$ e $42 \mathrm{~mm}$, nas mesmas condições. Além disso, os autores encontraram altura de $1,80 \mathrm{~m}$ e $41 \mathrm{~mm}$ de diâmetro para $S$. multijuga no modelo consorciado, uma vez que não houve sobreviventes para o modelo somente com o plantio de mudas de leguminosas arbóreas. Assim, os valores obtidos para os parâmetros de crescimento do presente estudo apresentaram-se superiores em relação aos anteriormente citados para as espécies em questão.

$\mathrm{O}$ incremento médio mensal em diâmetro $\left(\mathrm{IMM}_{\mathrm{D}}\right)$ para o plantio foi de 2,06 $\mathrm{mm}$.mês ${ }^{-1}$. Para o crescimento em altura, o incremento médio mensal $\left(\mathrm{IMM}_{\mathrm{H}}\right)$ foi de $5,75 \mathrm{~cm} \cdot \mathrm{mês}^{-1}$. As espécies $C$. speciosa e $T$. granulosa obtiveram os melhores desempenhos em relação ao incremento em diâmetro, apresentando $I M M_{D}$ de 5,98 e 5,32 mm.mês ${ }^{-1}$. No caso do incremento em altura, destacaram-se $S$. multijuga e $S$. parahyba, com $\mathrm{IMM}_{\mathrm{H}}$ correspondentes a 14,73 e 14,63 cm.mês ${ }^{-1}$ (Figura 2). 


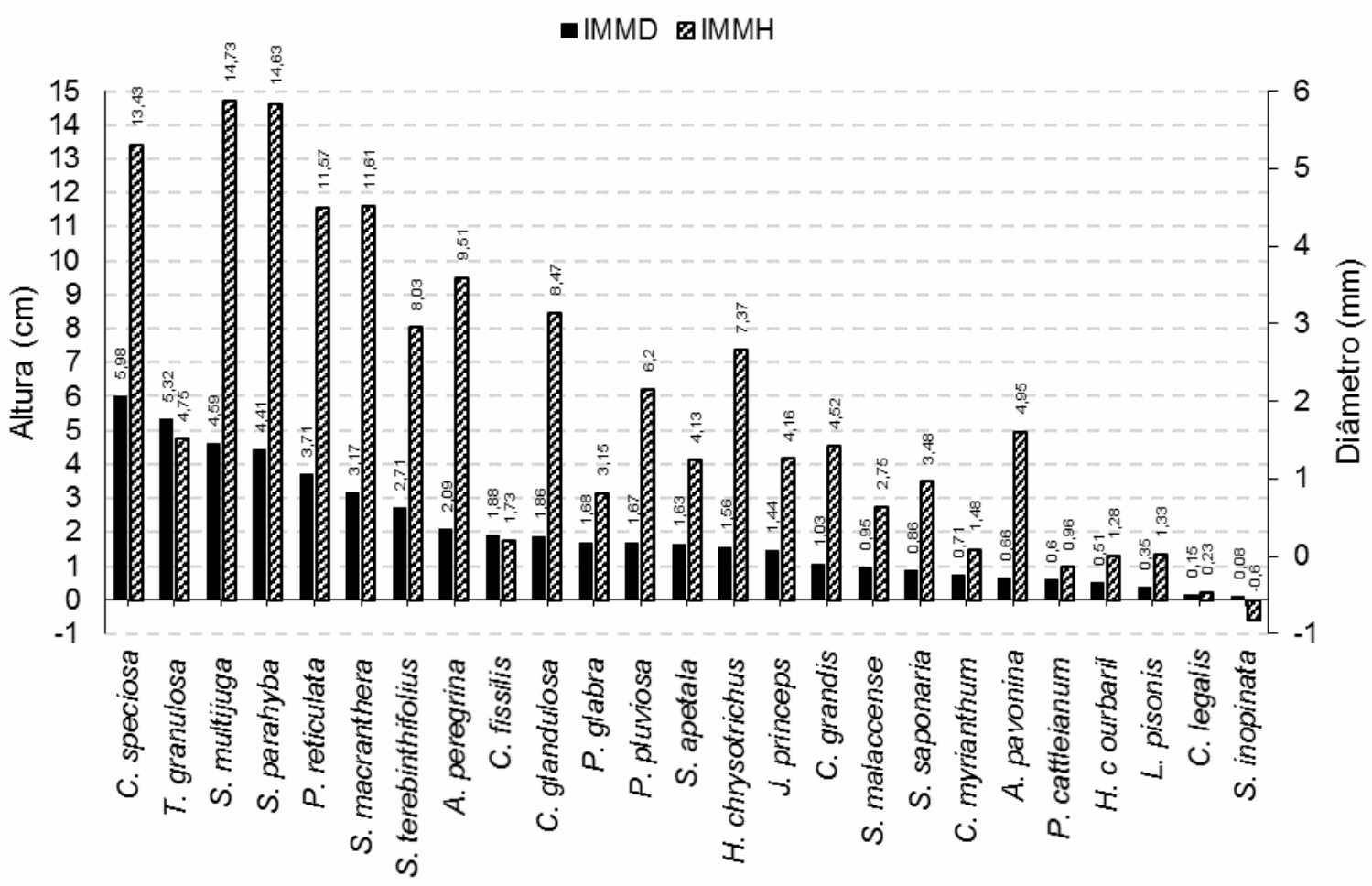

FIGURA 2: Incrementos Médios Mensais (IMM) para diâmetro (IMMD) e altura $\left(\mathrm{IMM}_{\mathrm{H}}\right)$ das espécies em crescimento na área de recuperação em Viçosa, MG.

No estudo conduzido por FRAGOSO et al. (2014) em plantios de enriquecimento no Paraná, a $C$. speciosa também obteve o maior incremento em diâmetro das espécies analisadas. As espécies $S$. multijuga e $P$. reticulata, além de se destacarem em termos de sobrevivência, obtiveram êxito no incremento em altura e diâmetro, com $I^{\prime M M} M_{H}$ de 14,73 e 11,57 cm.mês ${ }^{-1}$ e $I M M_{D}$ de 4,59 e 3,71 mm.mês ${ }^{-1}$, respectivamente. Já $S$. parahyba assumiu a décima primeira posição para o índice de sobrevivência, entretanto, se manteve entre as espécies que apresentaram os maiores incrementos para as variáveis analisadas.

Espécies como $P$. cattleianum, $H$. courbaril, $L$. pisonis, $C$. legalis e $S$. inopinata, não obtiveram incrementos expressivos das variáveis analisadas ao longo dos 20 meses. Esse fato pode ser decorrente da inadaptabilidade dessas espécies às condições submetidas no experimento. Em relação a $P$. cattleianum, MARCUZZO et al. (2015) também obtiveram taxa de incremento em diâmetro menor para a mesma, corroborando para a não recomendação da mesma, quando se objetiva um rápido recobrimento do solo.

$\mathrm{O} \mathrm{IMM}_{\mathrm{H}}$ negativo apresentado pela espécie $S$. inopinata ocorreu devido à alta incidência de quebra do caule das mudas dessa espécie. Esse fato provavelmente está relacionado à densidade utilizada no plantio, que é muito superior ao apresentado nas populações naturais da espécie, o que pode ter aumentado a probabilidade de ocorrência de injúrias nos indivíduos.

\section{CONCLUSÕES}

A sobrevivência do plantio apresenta-se heterogênea, tendo espécies com 95\% de sobrevivência e outras com $5 \%$. Isso indica que o conhecimento da adaptabilidade de espécies florestais para plantios de espécies nativas em áreas 
degradadas ainda precisa evoluir para melhorar a eficiência de projetos ligados a essa área.

As espécies $S$. terebinthifolius, $S$. saponaria, $P$. reticulata, $C$. grandis e $S$. multijuga são recomendadas para plantios mistos de recuperação de áreas degradadas em situações similares à do experimento. As espécies $P$. reticulata e $S$. multijuga, além do sucesso de sobrevivência, tiveram destaque nos parâmetros de crescimento.

O estudo possibilita inferir a respeito da potencialidade e restrição do uso de 25 espécies florestais no plantio de recuperação da área degradada na Mata Atlântica.

\section{REFERÊNCIAS}

ARRUDA, P. R. R.; SILVA, E.; COUTO, L. Problemática ambiental da Bacia Hidrográfica do Ribeirão São Bartolomeu, Viçosa-MG. Revista Árvore, v.23, p. 4953, 1999.

BRASIL. Lei no 9.985, de 18 de junho de 2000. Regulamenta o art. 225, § 1으, incisos I, II, III e VII da Constituição Federal, institui o Sistema Nacional de Unidades de Conservação da Natureza e dá outras providências. Disponível em: < http://www.planalto.gov.br/ccivil_03/leis/L9985.htm> Acesso em: 29 mar. 2016.

CARVALHO, P. E. R. Espécies arbóreas brasileiras. Brasília, DF: Embrapa Informação Tecnológica; Colombo: Embrapa Florestas, 2003. v. 1. (Coleção Espécies Arbóreas Brasileiras).

CAMPOE, O. C.; IANNELLI, C.; STAPE, J. L.; COOK, R. L.; MENDES, J. C. T.; VIVIAN, R. Atlantic forest tree species responses to silvicultural practices in a degraded pasture restoration plantation: From leaf physiology to survival and initial growth. Forest Ecology and Management, v.313, n. 1, p. 233-242, 2014. Disponível em: <http://dx.doi.org/10.1016/j.foreco.2013.11.016>. doi: 10.1016/j.foreco.2013.11.016

FERREIRA, W. C.; BOTELHO, S. A.; DAVIDE, A. C.; FARIA, J. M. R. Avaliação do crescimento do estrato arbóreo de área degradada revegetada à margem do Rio grande, na usina hidrelétrica de Camargos, MG. Revista Árvore, v.31, n.1, p.177185, 2007. Disponível em: <http://dx.doi.org/10.1590/S0100-67622007000100020> doi: 10.1590/S0100-67622007000100020

FUNDAÇÃO SOS MATA ATLÂNTICA. O Novo Código Florestal nos Estados da Mata Atlântica. Relatório técnico, 2016. Disponível em: < https://www.sosma.org.br/wp-content/uploads/2016/05/SOSMA-Estudo_CODIGOCAR_Final_ONLINE.pdf>. Acesso em: 20 mai. 2016.

FUNDAÇÃO SOS MATA ATLÂNTICA; INSTITUTO NACIONAL DE PESQUISAS ESPACIAIS. Atlas dos remanescentes florestais da mata atlântica período 20132014. Relatório técnico, São Paulo, 2015. Disponível em: < http://mapas.sosma.org.br/site_media/download/atlas_2013-

2014_relatorio_tecnico_2015.pdf> Acesso em: 20 mai. 2016.

FRAGOSO, R. O.; TEMPONI, L. G.; GUIMARÃES, A. T. B.; BONINI, K. Desenvolvimento de espécies arbóreas nativas em uma área reflorestada do 
corredor de biodiversidade Santa Maria-PR. Revista Árvore, v.38, n.6, p.1003-1013, 2014. Disponível em: http:<//dx.doi.org/10.1590/S0100-67622014000600005 >. doi: 10.1590/S0100-67622014000600005

MARCUZZO S. B.; ARAÚJO, M. M.; GASPARIN, E. plantio de espécies nativas para restauração de áreas em unidades de conservação: um estudo de caso no sul do Brasil. Floresta, v.45, n.1, p.129-140, 2015. Disponível em: <http://dx.doi.org/10.5380/rf.v45i1.32763>. doi: 10.5380/rf.v45i1.32763

MEIRA-NETO, J. A. A.; MARTINS, F. R. Composição florística de uma floresta estacional semidecidual montana no município de Viçosa-MG. Revista Árvore, v.26, n.4, p.437-446, 2002. Disponível em: <http://dx.doi.org/10.1590/S010067622002000400006>. doi: 10.1590/S0100-67622002000400006

MORAES, L. F. D.; ASSUMPÇÃO, J. M.; LUCHIARI, C; PEREIRA, T. S. Plantio de espécies arbóreas nativas para a restauração ecológica na Reserva Biológica de Poço das Antas, Rio de Janeiro, Brasil. Rodriguésia, v.57, n.3, p. 477-489. 2006.

OLIVEIRA, M. C.; RIBEIRO, J. F.; PASSOS. F. B.; AQUINO, F. G.; OLIVEIRA, F. F.; SOUSA, S. R. Crescimento de espécies nativas em um plantio de recuperação de Cerrado sentido restrito no Distrito Federal, Brasil. Revista Brasileira de Biociências, v.13, n.1, p. 25-32, 2015.

PAIVA, A.V.; POGGIANI, F. Crescimento de mudas de espécies arbóreas nativas plantadas no sub-bosque de um fragmento florestal. Scientia Forestalis, v.?, n.57, p. 141-151, 2000.

PEREIRA, J. S.; ABREU, C. F. N. R.; PEREIRA JUNIOR, R. A.; RODRIGUES, S. C. Avaliação do índice de sobrevivência e crescimento de espécies arbóreas utilizadas na recuperação de área degradada. Revista Geonorte, Edição Especial, v.1, n.4, p.138-148, 2012.

QUEIROZ, S. E. E.; PELOSI, A. P.; SILVA, L.V.; PEREIRA JUNIOR, A. M. Efeito do protetor físico na semeadura direta sobre o crescimento de quatro espécies arbóreas. Enciclopédia Biosfera, v. 9, n.17, p. 1193, 2013.

RESENDE, L. A.; PINTO, L. V. A.; SANTOS, E. C.; SILVA, S. Crescimento e sobrevivência de espécies arbóreas em diferentes modelos de plantio na recuperação de área degradada por disposição de resíduos sólidos urbanos. Revista Árvore, v.39, n.1, p.147-157, 2015. Disponível em: <http://dx.doi.org/10.1590/0100-67622015000100014>. doi: 10.1590/010067622015000100014

TABARELli, M.; AgUiAR A. V.; RiBeiRo M. C.; MetZGeR, J. P.; Peres, C. A. Prospects for biodiversity conservation in the Atlantic Forest: lessons from aging human-modified landscapes. Biological Conservation, v.143, n. 10, p. 2328-2340, 2010. Disponível em: <http://dx.doi.org/10.1016/j.biocon.2010.02.005>. doi:10.1016/j.biocon.2010.02.005 
UNIVERSIDADE FEDERAL DE VIÇOSA - UFV. Departamento de Engenharia Agrícola. Estação Climatológica Principal de Viçosa. Boletim meteorológico 2016. Viçosa, 2016. 